Check for updates

Cite this: Nanoscale, 2018, 10, 19272

Received 13th August 2018,

Accepted 24th September 2018

DOI: $10.1039 / c 8 n r 06536 e$

rsc.li/nanoscale

\section{EurOgels: A ferromagnetic semiconductor with a porous structure prepared via the assembly of hybrid nanorods $\uparrow$}

\author{
Bastian Trepka, Julian Stiegeler, Ilona Wimmer, Mikhail Fonin (iD and \\ Sebastian Polarz (D) *
}

EuO is unique, because it belongs to the few solids combining semiconducting properties $\left(E_{\text {gap }}=1.1 \mathrm{eV}\right)$ with native ferromagnetism. For future applications of EuO, e.g. as spin-filters or for sensors, one has to learn how defined nanostructures can be prepared. Unlike other ceramic oxides, there are no established softchemistry routes (e.g. sol-gel) towards EuO nanomaterials e.g. porous materials. This is due to the labile nature of the oxidation state $\mathrm{Eu}(+\mathrm{II})$. We present a particle-based method leading to a EuO aerogel. Instead of making the target material directly, we use nanoparticles of an organic-inorganic hybrid phase $\left(\mathrm{Eu}_{2} \mathrm{O}_{3}-\right.$ benzoate) and assemble those into an aerogel, followed by the transformation into phase-pure EuO. It is shown that organic aldehydes act as capping agents for controlling the morphogenesis of the hybrid particles. Depending on the steric demand of the aldehyde, one obtains plate-like particles or nanorods with increasing aspect ratio. The particles form a gel, when the aspect ratio is increased to $>20$. After supercritical drying, one receives a nanorod-based aerogel. Treatment of the latter with Eu-vapor leads to reduction of the $\mathrm{Eu}_{2} \mathrm{O}_{3}$ domains to $\mathrm{EuO}$ while retaining the aerogel structure. Proof of ferromagnetism in the resulting EuO aerogel was delivered by SQUID measurements.

A major promise in particle-based materials is cooperative effects and, thus, new features may emerge from the controlled assembly of nanoparticles in 3-D. Materials with open porosity are attractive, because this gives access to the surfaces of the nanoparticles and their properties. Therefore, researchers have tried to construct low-density and highly porous networks from functional nanoparticles. ${ }^{1}$ Lowest densities are realized for the so-called aerogels. An aerogel can be obtained via full preservation of the network structure of a solvent-filled gel during drying. The majority of known aerogels are prepared via a sol-gel process using molecular precursors followed by

University of Konstanz, Universitaetsstrasse 10, 78457 Konstanz, Germany. E-mail: sebastian.polarz@uni-konstanz.de

$\dagger$ Electronic supplementary information (ESI) available: Experimental details and additional analytical data. See DOI: 10.1039/c8nr06536e supercritical drying. However, with the availability of defined nanoparticles, one became interested in using them as building blocks for aerogels. The field has recently been reviewed by Eychmüller, ${ }^{2}$ who is also among the pioneers in this field. A focus of current research is on semiconductor quantum dots as building blocks and their optical properties. ${ }^{3}$ For instance Bigall et al. have described in 2015 an aerogel comprising II/VI semiconductor nanorods with remarkable optoelectronic properties. ${ }^{4}$ In 2016 Niederberger et al. assembled aerogels from $\mathrm{Y}_{2} \mathrm{O}_{3}$ nanosheets and demonstrated besides phosphorescense properties the possibilities for further modification of the yttria surfaces. ${ }^{5}$ Much less is known about particle-based, magnetic aerogels. Because of the availability of ferrite-based nanoparticles and ferrofluids, some papers can be found using them as building-blocks. For instance, Berglund and coworkers have used freeze-dried cellulose as a template for the deposition of ferrite nanoparticles. ${ }^{6}$ However, the cellulose matrix was not removed and, thus, remained as an integral part of the material.

Here, we aim at an aerogel made of a very special, magnetic material: EuO. Europium oxide with Eu in oxidation state (+II) is special, because it represents one of the few cases of a semiconductor possessing native ferromagnetism. The band gap of $\mathrm{EuO}\left(E_{\text {gap }}=1.1 \mathrm{eV}\right)$ is similar to that of silicon and it can be described as an almost ideal Heisenberg ferromagnet with a high magnetic moment of $7 \mu_{\mathrm{B}}$ per $\mathrm{Eu}^{\mathrm{II}}$ and a Curie temperature $T_{\mathrm{C}}=69 \mathrm{~K}^{7} \mathrm{EuO}$ is a half metal with the spin polarization approaching $100 \%,{ }^{7}$ making it an ideal candidate for spintronic applications, like spin filtering. ${ }^{8}$ There are only very few reports about EuO nanomaterials. ${ }^{9-11}$ Low-temperature, softchemistry methods like sol-gel processing fail because of the reducing character of $\mathrm{Eu}^{2+}\left(U_{\mathrm{Eu}^{\mathrm{I}} / \mathrm{Eu}^{\mathrm{III}}}=-0.35 \mathrm{~V}\right)$, which is sufficient to react with water resulting in $\mathrm{Eu}_{2} \mathrm{O}_{3} \cdot{ }^{12}$ Further difficulties arise due to the need for a very precise control of stoichiometry. Recently, we presented a new way for the preparation of EuO nanostructures. ${ }^{13}$ Instead of the preparation of the EuO material directly, an organic-inorganic hybrid material was generated first. The organic-inorganic phase 
resulted from the solvothermal conversion of $\mathrm{Eu}(\mathrm{ONap})_{2}$ (Nap $\cong$ naphtyl) as a precursor with benzyl alcohol $(\mathrm{BnOH})$ as a solvent. In agreement with a mechanism revealed by others for Yttrium-based materials, ${ }^{14} \mathrm{BnOH}$ becomes oxidized to benzoic acid $(\mathrm{BnOOH})$, which then forms a coordination polymer with the metal cation. The resulting $\mathrm{Eu}_{2} \mathrm{O}_{3}$-benzoate was forming hollow nanotubes (see also ESI Fig. S1 $\dagger$ ). ${ }^{13}$ We also showed, in a second step, that the conversion to EuO is possible while retaining the nanotube morphology. The described hollow nanotube architecture was discovered by accident. Here, we want to systemize the approach and access a broader variety of EuO nanostructures by controlling the shape of the organic-inorganic hybrid phase.

\section{Morphology control of nanoparticles}

A known approach to control the growth of crystalline particles is the addition of capping agents, ligands binding selectively to certain facets. The two candidates for ("intrinsic") capping agents responsible for the formation of the hollow nanotubes in ref.13 are obviously either $\mathrm{BnOH}$ or naphtolate (ONap), or both. For changing the morphology of the $\mathrm{Eu}_{2} \mathrm{O}_{3}$-benzoate phase, we used 4-isopropyl benzyl alcohol or 4-tertbutyl-benzyl alcohol instead of $\mathrm{BnOH}$ during the solvothermal process (Fig. S2 $\dagger$ ), and $\mathrm{Eu}(\mathrm{BPhen})_{2}$ (BPhen $\cong 2,6$-di $^{\text {tert }}$ butyl phenolate) as a precursor instead of $\mathrm{Eu}(\mathrm{ONap})_{2}$ (Fig. S3†). The morphology changes in both cases. There seems to be a preference for nanotube growth. However, the resulting particle dispersions are poor regarding uniformity in shape and size. Therefore, we tested, if an improvement was possible by the addition of extra capping agents. Compared to the reference case (ref.13) there are only minor effects for ligands containing $-\mathrm{COO}^{-}, \mathrm{S}, \mathrm{N}$, or $\mathrm{P}$ (Fig. S4 $\dagger$ ). Therefore, we assume that these substances are "inactive" and do not sufficiently interact with the surfaces of the $\mathrm{Eu}_{2} \mathrm{O}_{3}$-benzoate phase. Interestingly, benzyl aldehyde (BA) has a marked effect (Fig. $\mathrm{S} 5 \dagger$ ). $\mathrm{BA}$ is able to restrict the growth of the benzoate $\mathrm{Eu}_{2} \mathrm{O}_{3}$ phase in two dimensions, when used at low concentration. By transmission electron microscopy (TEM), one sees rod-like particles. The dark stripes are caused by the higher contrast of the $\mathrm{Eu}_{2} \mathrm{O}_{3}$ domains. The thickness of these domains is $1.3 \mathrm{~nm}$, which fits to one single unit cell $\left(a\left(\right.\right.$ monocl $\left.\left.\cdot \mathrm{Eu}_{2} \mathrm{O}_{3}\right)=1.4 \mathrm{~nm}\right)$. At higher amounts of $\mathrm{BA}$, there is a change in morphology to thin plates (Fig. S5†). Encouraged by the latter result, we tested, if other aldehydes can also act as capping agents. This is clearly the case as can be seen from Fig. 1. Magnified images can be found in Fig. S6. $\dagger$ There is an interesting trend. The more sterically demanding the aldehyde becomes, the longer the nanorods get ( $n$-butyl aldehyde < cyclohexyl aldehyde $<$ isobutyl aldehyde $\approx$ benzyl aldehyde $<n$-decyl aldehyde). The aspect ratio of the nanoparticles grown in the presence of $n$-butyl aldehyde is only $\approx 2$. For $n$-decyl aldehyde as a capping agent, one can see rods up to $200 \mathrm{~nm}$ long. Interestingly, the width of the particles $(\approx 9 \mathrm{~nm})$ remains unchanged. Because benzoic acid binds to the (100) atomic planes, ${ }^{13}$ it seems that the alde-

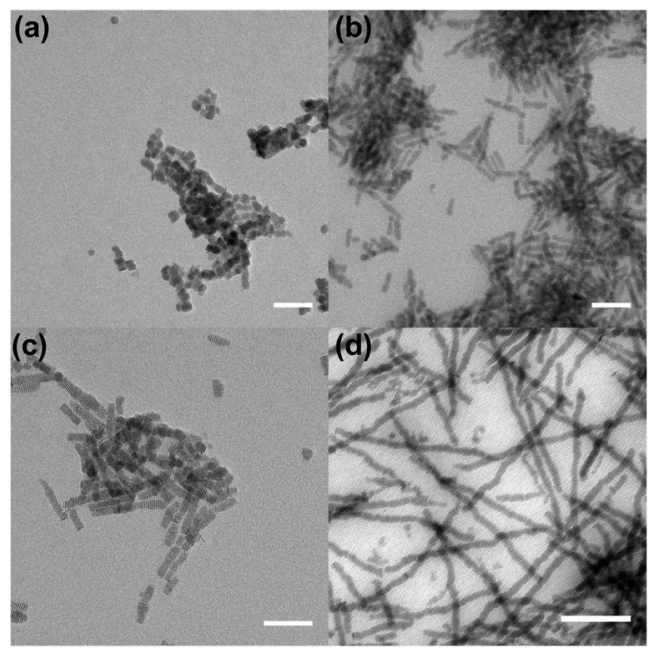

Fig. 1 TEM micrographs of $\mathrm{Eu}_{2} \mathrm{O}_{3}$-benzoate particles stabilized by $n$-butyl aldehyde (a; scalebar $50 \mathrm{~nm}$ ), cyclohexyl aldehyde (b; scalebar $50 \mathrm{~nm}$ ), isobutyl aldehyde (c; scalebar) and $n$-decyl aldehyde (d; scalebar $100 \mathrm{~nm}$ ) as capping agents.

hydes lead to a stabilization of the orthogonal directions (e.g. (010), (001)).

\section{Hybrid aerogel}

It is well documented in the literature that a threshold exists for elongated colloidal objects, when percolation occurs. ${ }^{15}$ Thus, gelation can occur, if the length of the colloids is sufficient. This is the case for the particles prepared using $n$-decyl aldehyde (DA) as a capping agent (Fig. 2a). The wet gel can be converted to an aerogel by extraction and supercritical drying with $\mathrm{CO}_{2}$ (Fig. 2b).

The structure of the aerogel was analyzed by a combination of methods. The powder X-ray diffraction pattern (Fig. 3a) can be explained by a superposition of a lamellar structure with a periodicity of $1.8 \mathrm{~nm}$ and monoclinic $\mathrm{Eu}_{2} \mathrm{O}_{3}$. This structure is confirmed by TEM (Fig. 3e) and it is also indicated that the aerogel still contains the nanorods. See further proof by scan-

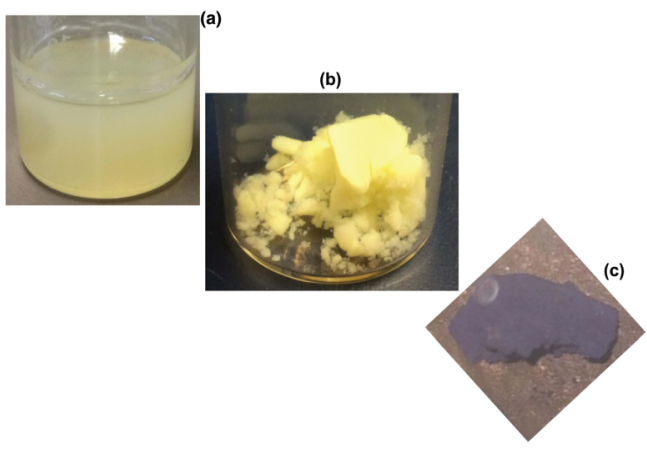

Fig. 2 Photographic images of a gelated dispersion of $\mathrm{Eu}_{2} \mathrm{O}_{3}$-benzoate nanorods grown in the presence of DA (a), the aerogel obtained after supercritical drying (b) and the aerogel after conversion to EuO (c). 


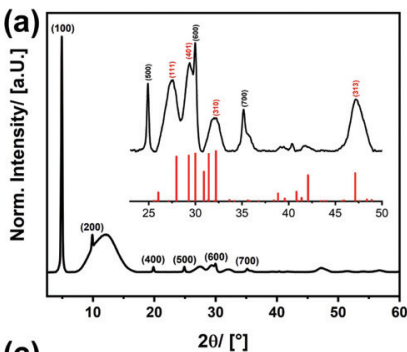

(c)

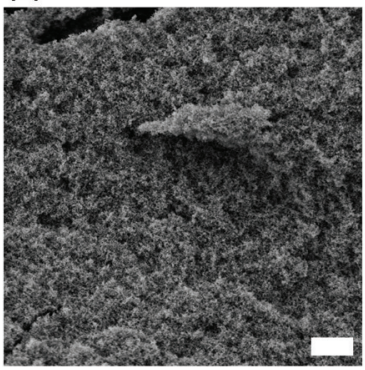

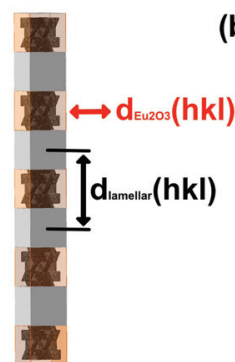

(b)

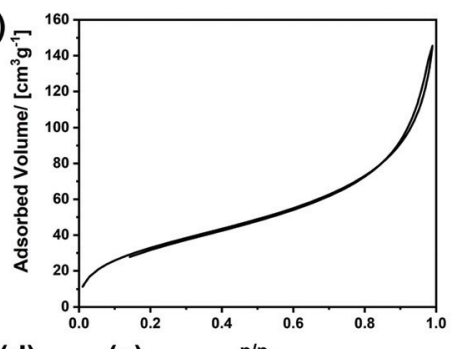

(d)

(e)

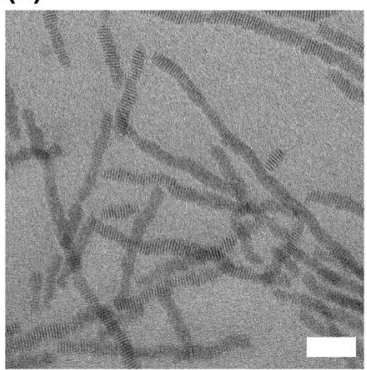

Fig. 3 Nanostructure investigation of the particle-based, benzoate- $\mathrm{Eu}_{2} \mathrm{O}_{3}$ hybrid aerogel. PXRD and model for the structure of an individual nanorod (a). The inset also shows the reference pattern of monoclinic $\mathrm{Eu}_{2} \mathrm{O}_{3}$ (ICSD-8056). $\mathrm{N}_{2}$ physisorption adsorption-desorption isotherm (b), SEM (c, scalebar $20 \mu \mathrm{m}$; d, scalebar $200 \mathrm{~nm}$ ) and TEM (e, scalebar $25 \mathrm{~nm}$ ).

ning electron microscopy (SEM, Fig. 3d). The presence of benzoate was confirmed by FT-IR spectroscopy (see Fig. S7†). There are no signals corresponding to DA, which indicates that it has been removed from the surfaces during the supercritical extraction procedure. The high porosity of the material is confirmed by SEM (Fig. 3c and d) as well as by $\mathrm{N}_{2}$ physisorption measurements. Evaluation of the isotherm (Fig. 3b) gives a specific surface area of $127.3 \mathrm{~m}^{2} \mathrm{~g}^{-1}$ and a broad pore-size distribution function, which is typical of aerogel materials. The yellow color results from an adsorption band at $416 \mathrm{~nm}$ and the photoluminescence properties are in agreement with those of $\mathrm{Eu}^{\mathrm{III}}$ in an asymmetric ligand field (Fig. S7†). The material is paramagnetic, which is in-line with the existence of magnetically decoupled Eu ${ }^{\mathrm{III}}$ centers (Fig. S7†).

\section{EuO aerogel}

Because we showed earlier, ${ }^{13}$ a benzoate- $\mathrm{Eu}_{2} \mathrm{O}_{3}$ hybrid can be converted to EuO by treatment with Eu-vapor, we hoped this would work for the presented aerogel material as well. The material was treated accordingly (see the experimental part given in the ESI $\dagger$ ). One obtains monolithic pieces with greyish color (Fig. 2c). The color of the aerogel results from the bandedge absorption of EuO ( $E_{\text {gap }}=1.14 \mathrm{eV}$; see Fig. S8†), which is in excellent agreement with the value of bulk EuO. ${ }^{7}$ For further proof, phase-pure EuO in the rock-salt structure (space group $F m \overline{3} \mathrm{~m}$ ) has been obtained which comes from PXRD measurements shown in Fig. 4a and high-resolution (HR)-TEM/electron diffraction (ED) (Fig. S8 †). SEM measurements (Fig. 4b-d) show that the aerogel structure could be retained. Compared to the benzoate- $\mathrm{Eu}_{2} \mathrm{O}_{3}$ aerogel, the material is no longer constructed from nanorods. This is caused by sintering processes, which are unavoidable considering the high temperatures needed for the reduction with Eu-vapor $\left(T=450{ }^{\circ} \mathrm{C}\right)$. Because of the sintering processes, the material has compacted to approximately half of its previous volume. As a further consequence of the sintering processes, the mechanical stability has changed as well. After reduction, the material seems to be harder but also more brittle compared to the aerogel prior to reduction. $\mathrm{N}_{2}$ physisorption analysis (Fig. 4e) shows that the EuO aerogel is a macroporous solid with highly accessible pores and a reasonable surface area of $15 \mathrm{~m}^{2} \mathrm{~g}^{-1}$. This value might appear low at first sight, because aerogels with a much higher surface are known in the literature for other materials like $\mathrm{SiO}_{2}$. However, one also has to consider that the density of $\mathrm{EuO}\left(\rho=8.2 \mathrm{~g} \mathrm{~cm}^{-3}\right)$ is much higher than that of e.g. $\mathrm{SiO}_{2}((\rho \approx$ $\left.2.5 \mathrm{~g} \mathrm{~cm}^{-3}\right)$. Unfortunately, the reduction with $\mathrm{Eu}$ vapor requires higher temperatures; thus, a certain degree of sintering is unavoidable leading to the reduction of the surface area. Thus, ongoing research is dealing with the development of an advanced reduction setup. Higher vacuum as well as more precise control over the europium vaporization is needed. Magnetic properties were investigated using superconducting quantum interference device (SQUID) measurements. The $T$-dependent measurements show a Curie point at $T=69 \mathrm{~K}$, which is the characteristic value for EuO (see Fig. S8†). ${ }^{16}$ Fielddependent measurements are shown in Fig. 4f and $\mathrm{g}$ for two temperatures below $T_{\mathrm{C}}$. The resulting curves exhibit the features, which are typical of a ferromagnetic material. Magnetization saturated at external fields below $2 \mathrm{~T}$. In general, the magnetic hysteresis is small even for bulk EuO. ${ }^{7}$ Therefore, the important area in the magnetization curves is enlarged in Fig. 4g. It can be seen that coercivity becomes significantly smaller with increasing the measurement temperature from 1.8 to $20 \mathrm{~K}$. For bulk EuO, this should not be 
(a)

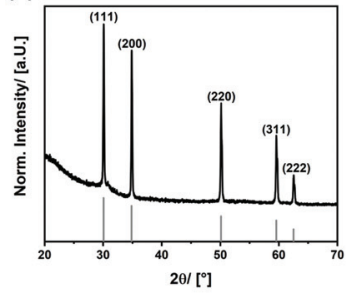

(f)

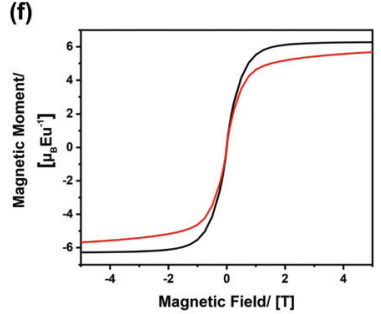

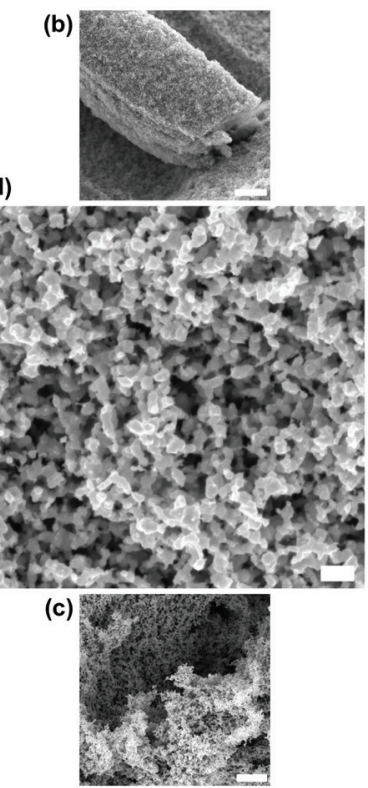
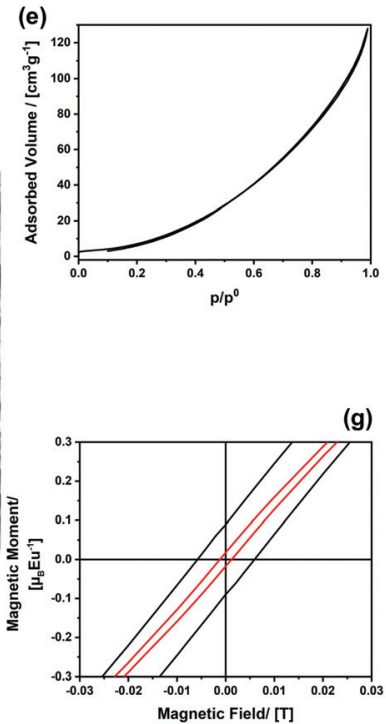

Fig. 4 Nanostructure investigation of the EuO aerogel. (a). PXRD pattern (black) and that of bulk EuO as a reference (grey bars). SEM micrographs taken at different magnification (b, scalebar $10 \mu \mathrm{m}$; c, scalebar $10 \mu \mathrm{m}$; d, scalebar $300 \mathrm{~nm}$ ). (e) $\mathrm{N}_{2}$ physisorption adsorption-desorption isotherm. SQUID measurements (black: $1.8 \mathrm{~K}$; red: $20 \mathrm{~K}$ ) (f) and magnification of the hysteresis area (g).

the case, because both temperatures are below $T_{\mathrm{C}}$. However, the EuO aerogel is constructed from a network of nanosized crystallites with sizes ranging from $20 \mathrm{~nm}$ to $\approx 50 \mathrm{~nm}$ (Fig. $4 \mathrm{~d}$ ). Ferromagnetic particles become superparamagnetic below a certain size, and the associated blocking temperature is size dependent. Therefore, it seems that at $20 \mathrm{~K}$ a certain fraction of the EuO particles in the aerogel have already switched to the superparamagnetic state, resp. for them the blocking temperature is below $20 \mathrm{~K}$.

\section{Conclusions}

Here, we have presented not only one of the first cases for a ferromagnetic aerogel, but the material is a semiconductor at the same time. A EuO aerogel could be prepared by a particlebased approach. Aided by organic aldehydes as capping agents, it was possible to increase the aspect ratio of organic-inorganic hybrid $\mathrm{Eu}_{2} \mathrm{O}_{3}$-benzoate nanorods sufficiently for inducing gelation. After supercritical drying and reduction using Eu-vapor, the final phase-pure EuO material was obtained. The received material profile could be interesting for applications like spin filtering, sensing or magnetic purification. Furthermore, infiltration of the macroporous structure with e.g. different types of functional nanoparticles is thinkable. This could open a complete new pathway for the creation of highly functional $\mathrm{EuO} / \mathrm{Metal}$ or EuO/Semiconductor heterostructures.

\section{Conflicts of interest}

There are no conflicts to declare.

\section{Acknowledgements}

The authors gratefully acknowledge financial support from the German Research Foundation (DFG) via SFB1214 Project A5. The authors gratefully acknowledge technical/instrumental support from the German Research Foundation (DFG) via SFB1214 Project Z1 Particle Analysis Center.

\section{Notes and references}

1 L. Cademartiri and K. J. M. Bishop, Nat. Mater., 2015, 14, 2-9.

2 C. Ziegler, A. Wolf, W. Liu, A. K. Herrmann, N. Gaponik and A. Eychmüller, Angew. Chem., Int. Ed., 2017, 56, 1320013221.

3 B. Cai, V. Sayevich, N. Gaponik and A. Eychmüller, Adv. Mater., 2018, 1707518.

4 S. Sánchez-Paradinas, D. Dorfs, S. Friebe, A. Freytag, A. Wolf and N. C. Bigall, Adv. Mater., 2015, 27, 61526156.

5 W. Cheng, F. Rechberger and M. Niederberger, ACS Nano, 2016, 10, 2467-2475.

6 R. T. Olsson, M. A. S. Azizi Samir, G. Salazar-Alvarez, L. Belova, V. Ström, L. A. Berglund, O. Ikkala, J. Nogués and U. W. Gedde, Nat. Nanotechnol., 2010, 5, 584.

7 A. Schmehl, V. Vaithyanathan, A. Herrnberger, S. Thiel, C. Richter, M. Liberati, T. Heeg, M. Rockerath, L. F. Kourkoutis, S. Muhlbauer, P. Boni, D. A. Muller, Y. Barash, J. Schubert, Y. Idzerda, J. Mannhart and D. G. Schlom, Nat. Mater., 2007, 6, 882-887. 
8 C. Caspers, A. Gloskovskii, M. Gorgoi, C. Besson, M. Luysberg, K. Z. Rushchanskii, M. Ležaić, C. S. Fadley, W. Drube and M. Müller, Sci. Rep., 2016, 6, 22912.

9 T. Mairoser, J. A. Mundy, A. Melville, D. Hodash, P. Cueva, R. Held, A. Glavic, J. Schubert, D. A. Muller, D. G. Schlom and A. Schmehl, Nat. Commun., 2015, 6, 7716.

10 Y. Hasegawa, S. Thongchant, Y. Wada, H. Tanaka, T. Kawai, T. Sakata, H. Mori and S. Yanagida, Angew. Chem., Int. Ed., 2002, 41, 2073-2075.

11 S. Thongchant, Y. Hasegawa, Y. Wada and S. Yanagida, Chem. Lett., 2001, 1274-1275.
12 M. J. Bierman, K. M. Van Heuvelen, D. Schmeißer, T. C. Brunold and S. Jin, Adv. Mater., 2007, 19, 2677-2681.

13 B. Trepka, P. Erler, S. Selzer, T. Kollek, K. Boldt, M. Fonin, U. Nowak, D. Wolf, A. Lubk and S. Polarz, Adv. Mater., 2018, 30, 7.

14 N. Pinna, G. Garnweitner, P. Beato, M. Niederberger and M. Antonietti, Small, 2005, 1, 112-121.

15 S. Tanja, A. M. Mark and S. Paul van der, Europhys. Lett., 2015, 111, 56004.

16 T. R. McGuire and M. W. Shafer, J. Appl. Phys., 1964, 35, 984-988. 